\title{
GENERATORS OF THE RING OF BOUNDED OPERATORS
}

\section{CHANDLER DAVIS}

J. A. Dieudonné suggested in conversation that some small number of projections might suffice to generate the ring $\mathbb{B}$ of all bounded operator on separable Hilbert space $\mathfrak{H C}$. There is some analogy between such a result and the theorem that a compact connected metric group can be generated by two elements. ${ }^{1}$ The analogy is still closer for Theorem 2 below.

In this paper, operators $A, \ldots$ will be said to "generate" the smallest ring (i.e., weakly closed self-adjoint algebra) containing $A, \cdots$ and the constants.

THEOREM 1. There exist three projections which generate the ring $\mathbb{B}$. The number three cannot be reduced if $\mathfrak{H C}_{\mathrm{C}}$ has dimensionality 3 or greater.

If $\operatorname{dim}(\mathfrak{H C})=1$, there is nothing but constants in $\mathscr{B}$. If $\operatorname{dim}(\mathfrak{C})=2$, then any two noncommuting projections generate $\mathbb{B}$. Hereafter suppose $\operatorname{dim}(\mathfrak{H C}) \geqq 3$.

I will use what I shall call the closeness operator $C=C(E, F)$ associated with any two projections $E, F$. It is defined by $C(E, F)=1-E$ $-F+E F+F E=E F E+(1-E)(1-F)(1-E)$. It is a positive definite operator which, in case ${ }^{2} E \cap F+E \cap(1-F)+(1-E) \cap F+(1-E)$ $\cap(1-F)=0$, acts like "the square of the cosine of the angle" between $E$ JC and FHe.

To show $E$ and $F$ fail to generate $\mathcal{B}$, I shall show some nonconstant operator commutes with both; this is enough because such an operator commutes with the whole ring generated by $E$ and $F$, whereas the commutator of $\mathcal{B}$ contains only constants. Since $C(E, F)$ commutes with $E$ and $F$, the only case to be considered is $C$ constant. $E \neq 0$ may be assumed. Choose $x=E x, x \neq 0, x$ otherwise arbitrary. Now the subspace spanned by $x$ and $F x$ is not zero, and since it is at most 2-dimensional it is not $\mathfrak{F C}$; so the projection $P$ on it is nonconstant. $P \mathcal{H}$ is invariant under $E$ and $F$, since $E F x=E F E x=C x$, which in this case is a multiple of $x$. Therefore $P$ is a nonconstant operator

Received by the editors September 24, 1954 and, in revised form, January 7, 1955.

${ }^{1} \mathrm{H}$. Auerbach, Sur les groupes linéaires bornées (III), Studia Mathematica vol. 5 (1934) pp. 43-49. J. Schreier and S. Ulam, Sur le nombre des génerateurs d'un groupe topologique compact et connexe, Fund. Math. vol. 24 (1935) pp. 302-304.

2 Here " $\cap$ " is intersection in the lattice of projections. This equation says EJC and FJC are in position $p$ (J. Dixmier, Position relative de deux varietés linéaires fermées dans un espace de Hilbert, Rev. Sci. vol. 86 (1948) pp. 387-399). 
commuting with $E$ and $F$.

Now for the proof of the first sentence in the theorem. Only countable dimensionality will be treated, finite dimensionality is handled similarly. There is a good deal of leeway in the construction; the particular generators $E_{1}, E_{2}, E_{3}$ given here are chosen for convenience.

Let $x_{1}, y_{1}, x_{2}, y_{2}, x_{3}, \cdots$ be an orthonormal basis of $\mathfrak{H}$. Let $z_{n}=\cos \theta_{n} x_{n}+\sin \theta_{n} y_{n}, n=1,2, \cdots$, with $\theta_{n}=\pi /(2 n+1)$. Let $P_{n}$ be the projection on $\left[x_{n}, y_{n}\right]$, the subspace spanned by $x_{n}$ and $y_{n}$, $n=1,2, \cdots$. Let $E_{1}$ be the projection on $\left[x_{1}, x_{2}, x_{3}, \cdots\right] ; E_{2}$, the projection on $\left[z_{1}, z_{2}, z_{3}, \cdots\right]$.

Now the ring generated by $E_{1}$ and $E_{2}$ contains $C=C\left(E_{1}, E_{2}\right)$. It can be shown by a direct computation that the eigenspaces of $C$ are the $P_{n} \mathfrak{H C}$, the corresponding eigenvalues being $\cos ^{2} \theta_{n}$. Each eigenvalue is an isolated point of the spectrum; the characteristic function of the set containing only $\cos ^{2} \theta_{n}$ is measurable (even continuous) on the spectrum of $C$. The spectral theorem implies that the ring contains all the $P_{n}$. Also the ring contains every operator on $P_{n} \mathcal{H C}$, for on that 2-dimensional Hilbert space $E_{1}$ and $E_{2}$ are noncommuting projections (see the remark at the beginning of the proof).

Finally, define $E_{3}$ as the projection on

$$
\left[x_{1}+x_{2}, y_{2}+y_{3}, \cdots, x_{2 n-1}+x_{2 n}, y_{2 n}+y_{2 n+1}, \cdots\right] .
$$

The ring $R$ generated by $E_{1}, E_{2}$, and $E_{3}$ will be shown to be $\mathbb{B}$.

Let $E(x ; y)$ denote, for any unit vectors $x$ and $y$, the operator characterized by

$$
\begin{gathered}
E(x ; y) x=y \\
z \perp x \quad \text { implies } \quad E(x ; y) z=0 .
\end{gathered}
$$

Also hereafter let $w_{n}$ mean either $x_{n}$ or $y_{n}, n=1,2, \cdots$.

$R$ contains $E\left(x_{2 n-1} ; x_{2 n}\right)$. For it contains the projection on $\left[x_{2 n-1}\right]$, and premultiplying that projection by $2 P_{2 n} E_{3}$ gives the desired operator. Therefore $R$ contains $E\left(w_{2 n-1} ; w_{2 n}\right)$, and necessarily also its adjoint, $E\left(w_{2 n} ; w_{2 n-1}\right)$. Similarly, $R$ contains $E\left(y_{2 n} ; y_{2 n+1}\right)$, hence $E\left(w_{2 n} ; w_{2 n+1}\right)$ and $E\left(w_{2 n+1} ; w_{2 n}\right)$. By induction, $E\left(w_{i} ; w_{j}\right) \in R$. Therefore $R$ contains every operator whose matrix, using the originally given orthonormal basis of $\mathfrak{H}$, has finitely many nonzero entries.

Let $A \in \mathcal{B}$, and let

$$
A_{n}=\sum_{1}^{n} P_{k} A P_{k}
$$


Then $A_{n} \in \mathbb{R}$, and $A$ is the weak limit of the $A_{n}$, so $A \in R$. It has been proved that $R=B$.

(Weak closure of $R$ was required only in the last paragraph of the proof; uniform closure was all that was used before. I do not know if there exist three projections which generate $B$ by algebraic operations and uniform limits. ${ }^{8}$ )

THEOREM 2. There exist two unitary operators which generate $\mathcal{B}$. They may be chosen so one of them is a symmetry.

This will be proved relying largely on the previous proof, and keeping the same symbols. Again I shall treat only the countable case.

Let $U x_{n}=z_{n}, U y_{n}=-\sin \theta_{n} x_{n}+\cos \theta_{n} y_{n}$. This defines $U$ as a unitary operator. Let $V=1-2 E_{3}$, a symmetry. The ring $R^{\prime}$ generated by $U$ and $V$ will be shown to be $\mathbb{B}$.

$E_{3}=(1-V) / 2 \in R^{\prime}$. As above, one shows first that each $P_{n} \mathfrak{F C}$ is an eigenspace of $U+U^{*}$, corresponding to the eigenvalue $2 \cos \theta_{n}$; and therefore that $P_{n} \in R^{\prime}$. The last part of the previous proof can be invoked once it is shown that $R^{\prime}$ contains every operator on the 2dimensional subspace $P_{n} \mathcal{H C}$. But $2 P_{n} E_{3} P_{n} \in R^{\prime}$ and $2 U P_{n} E_{3} P_{n} U^{*} \in R^{\prime}$ are noncommuting projections operating on $P_{n} \mathfrak{H C}$.

\section{AnN Arbor, Michigan}

3 The projections given here do not. In fact, every operator $A$ which is a uniform limit of polynomials in $E_{1}, E_{2}$, and $E_{3}$, has the special property (among others) that $\left(A x_{2 n}, x_{2 n}\right)$ has a limit as $n \rightarrow \infty$. Proof omitted. 